\title{
Quasi-Optical Terahertz Polarizers Enabled by Inkjet Printing of Carbon Nanocomposites
}

Arindam Das,* Thomas Schutzius, Constantine M. Megaridis ${ }^{\text {a) }}$

Department of Mechanical and Industrial Engineering, University of Illinois at Chicago, Chicago, Illinois 60607

Subhali Subhechha,* Tao Wang, Lei Liu ${ }^{\text {b) }}$

Department of Electrical Engineering, University of Notre Dame, Notre Dame, Indiana 46556

\begin{abstract}
We report a new approach for cost-effective manufacturing of THz quasi-optical polarizers by inkjet printing of polymer-carbon nanowhisker (CNW) dispersions. The electromagnetic interference properties of coatings with fixed $\mathrm{CNW}$ /polymer composition and varying thickness are quantified by a frequency domain $\mathrm{THz}$ spectroscopy system in the range 570-630GHz. A shielding effectiveness of $\sim 40 \mathrm{~dB}$ is attained for $70 \mu \mathrm{m}$-thick coatings. A prototype $\mathrm{THz}$ polarizer printed on Mylar film displayed transmission and absorbance that varied with polarization orientation. The degree of polarization for film thickness of $\sim 1 \mu \mathrm{m}$ was 0.35 . This performance can be improved by refining grid dimensions, increasing coating thickness and adopting multilayer polarizer structures.
\end{abstract}

\footnotetext{
*These authors contributed equally to this work.

a) Electronic mail: cmm@uic.edu (C. M. Megaridis); ${ }^{\text {b) }}$ 1liu3@nd.edu (L. Liu)
} 
Electromagnetic waves in the frequency range $0.1-10 \mathrm{THz}$ have remained the least explored and developed in the entire spectrum, creating what is widely known as the Terahertz Gap. Recently, THz waves have attracted much attention and continuous interest owning to their prospective applications in many important fields, such as astronomy, chemical analysis, biological sensing, imaging, security screening, etc. ${ }^{1,2,3,4,5}$ Among many $\mathrm{THz}$ quasi-optical components under development, THz polarizers are in high demand in spectroscopy, polarization interferometry, polarimetric detection (e.g., polarization of Cosmic Microwave Background), and other areas. ${ }^{6}$

THz polarizers are highly anisotropic devices that present maximum transmission $\left(\mathrm{T}_{\perp}\right)$ with incident wave polarization perpendicular to the alignment axis, while having highest attenuation $\left(\mathrm{T}_{/ /}\right)$with polarization parallel to the alignment direction. Conventional $\mathrm{THz}$ polarizers have been realized by metal wire-grids, ${ }^{7}$ resulting in expensive, heavy and bulky components, not suitable for many practical applications. To overcome this problem, micro-strip grids have been produced on substrates (e.g., silicon, polymer) for $\mathrm{THz}$ polarizers, using special techniques, such as nanoimprinting lithography and chemical etching. ${ }^{8,9,10}$ In addition, polarizers based on highly aligned single-walled carbon nanotube (SWNT) films (either on substrates or free-standing) have been demonstrated to have promising performance in the THz region. ${ }^{11,12}$ However, the available approaches either require complex (i.e., costly) or chemically aggressive fabrication steps, or are hindered by limitations in carbon nanotube film formation, growth and transfer. Due to the above, existing approaches limit the choice of substrates and are frequently detrimental to the environment by generating hazardous waste. Therefore, innovations in materials and processes for cost-effective, environmentally friendly, scalable and potentially high performance $\mathrm{THz}$ 
quasi-optical components, such as polarizers, are of immense interest for a number of advanced technology applications.

In previous work, ${ }^{13}$ we demonstrated that large area carbon nanofiber (CNF)/ polytetrafluoroethylene (PTFE) polymer composite coatings display effective THz shielding and attenuation properties, in addition to being superhydrophobic (liquid droplet contact angle > $150^{\circ}$ ) and self-cleaning (liquid droplet roll-off angle $<10^{\circ}$ ). The coating attenuation level was tuned by varying CNF loading content, and a THz shielding effectiveness (SE) of $\sim 32 \mathrm{~dB}$ was measured in the frequency range of $570-630 \mathrm{GHz}$ for a $\mathrm{CNF} /$ polymer mass ratio of 1.1 ; the polymer matrix was a blend of poly(vinylidene fluoride) (PVDF) and poly(methyl methacrylate) (PMMA). The coating fabrication process reported ${ }^{13}$ is inherently low-cost, and all ingredients are commercially available. If these materials could be patterned using a ubiquitous low-cost method (e.g., inkjet-printing), one could bypass the complex photolithography, nanoimprinting, or chemical etching processes, and the cost-effective manufacture of flexible THz quasi-optical components, such as polarizers and filters, might be realizable. The idea of inkjet printing conductive inks to fabricate low-cost electrical components has been explored recently ${ }^{14,15}$ in the fabrication of radio frequency antennas. The inks employed in those works were based on metal nanoparticles that require an additional thermal step $\left(100-120^{\circ} \mathrm{C}\right)$ to produce the final product. In general, metal nanoparticle inks can produce highly conductive traces, ${ }^{15}$ but due to their higher concentrations of solids (which are necessary for depositing continuous, electrically conducting traces), metal nanoparticle inks are more vulnerable to deposit non-uniformities caused by the coffee-stain effect. ${ }^{16}$ Carbon fiber-based inks require considerably lower concentrations of solids for depositing conducting traces, as the large aspect ratio of fibers allows percolation at a lower threshold compared to the metal nanoparticle inks. ${ }^{13}$ In addition, non-spherical particles are far 
less susceptible to the coffee stain effect. ${ }^{17}$ The attractiveness of carbon fiber-based inks has been extolled in Ref. 15 and the references cited therein.

In this letter, we report the design and fabrication of quasi-optical $\mathrm{THz}$ polarizers using dispersions of a fluoroacrylic polymer with carbon nanowhiskers (CNW, i.e., short-length CNF) which are dispensed using standard inkjet printing procedures. First, the electromagnetic interference (EMI) shielding effectiveness of continuous (large-area) CNW composite coatings is characterized in the $570 \mathrm{GHz}-630 \mathrm{GHz}$ frequency range. The inkjet printing process of the polarizers is then presented, followed by measurement results for a prototype THz polarizer. An average degree of polarization of 0.35 is achieved for a CNW coating thickness of $\sim 1 \mu \mathrm{m}$. The performance of the polarizers, specifically the extinction ratio, can be improved further by increasing the coating thickness and adopting dual-layer structures.

An electromagnetic (EM) wave polarizer usually consists of well-aligned grids with a period much less than one wavelength, and ideally, the finer the grid, the better is the polarizer performance. However, in practice, a polarizer's performance (i.e., extinction ratio, $T_{\perp} / T_{/ /}$) is limited by the finite conductivity of the metal wires or microstrips forming the grids. For $\mathrm{THz}$ polarizers patterned from thin film materials, the shielding effectiveness of the corresponding thin film, which increases monotonically with conductivity, ${ }^{7}$ sets an absolute upper limit for the polarization efficiency that can be achieved. Consequently, the SE of uniform CNW composite coatings had to be examined first.

A commercially available fluoroacrylic co-polymer (PMC) from DuPont (Capstone ${ }^{\circledR}$ ST100) was used as-received in the form of a $20 \mathrm{wt} . \%$ dispersion in water. The CNWs (solid core, 110nm-170nm dia., 5-9 $\mu$ m length) were procured from Sigma-Aldrich, USA. Reagent grade formic acid, acetic acid and acetone were obtained from Sigma-Aldrich and used as received. For 
the continuous coatings, the CNWs were first dispersed in acetone and formic acid (2:3 mass ratio) and probe sonicated (Sonics ${ }^{\circledR}, 750 \mathrm{~W}$, High Intensity Ultrasonic Processor, $13 \mathrm{~mm}$ diameter tip at $70 \%$ amplitude) for 3 minutes. CNW concentration in the acetone/formic acid medium was fixed at $1 \mathrm{wt} . \%$. Finally, $20 \mathrm{wt} . \%$ PMC dispersion in water was gradually added to the suspension until the CNW/PMC mass ratio reached unity, from which point the suspension was bath sonicated for an additional 10 minutes. The final dispersions were applied onto cellulosic paper substrates by means of an airbrush atomizer (Paasche, VL siphon feed, 0.55mm spray nozzle). Spray cast films were dried at $90^{\circ} \mathrm{C}$ under a convective air space heater for 5 minutes. Although the dispersion, and thus the coating composition were fixed, coating thickness was varied by applying additional spray layers. The SE of four coatings was measured (respective average thicknesses $24 \mu \mathrm{m}, 42 \mu \mathrm{m}, 61 \mu \mathrm{m}$ and $70 \mu \mathrm{m}$ ), and compared with the base SE of the uncoated paper substrate. The electrical conductivities of the coatings were measured by the two-probe method, and found to be $118 \mathrm{~S} / \mathrm{m}, 147 \mathrm{~S} / \mathrm{m}, 209 \mathrm{~S} / \mathrm{m}$ and $386 \mathrm{~S} / \mathrm{m}$, respectively. The rising conductivity with coating thickness is attributed to the increased compactness of the thicker coatings, which were created by repeated spray-on layers. The prolonged drying time thus caused the dried solid phase to be less porous. Hence thicker coatings had reduced thickness per unit deposited solid mass and displayed disproportionately higher electrical conductivity.

Figure 1a shows a scanning electron micrograph of the surface structure of a CNW/PMC (1:1 mass ratio) coating. The presence of the percolated CNWs in the PMC matrix is apparent. The shielding effectiveness of the coatings in the 570-630 GHz frequency range was measured by a frequency domain terahertz spectroscopy instrument ${ }^{18}$ shown in Fig. 1b. The THz radiation was provided by a VDI (Virginia Diodes, Inc.) frequency extension module (FEM, or multiplier chain) which converted a microwave $(10-20 \mathrm{GHz})$ signal from a synthesizer to $\mathrm{THz}$ radiation, 
covering the range from 570-630 GHz. The average output power in this range was approximately $1 \mathrm{~mW}$. The $\mathrm{THz}$ energy was coupled to a zero-bias Schottky diode broadband detector $^{19}$ through four off-axis parabolic mirrors A-D (see Fig. 1b).
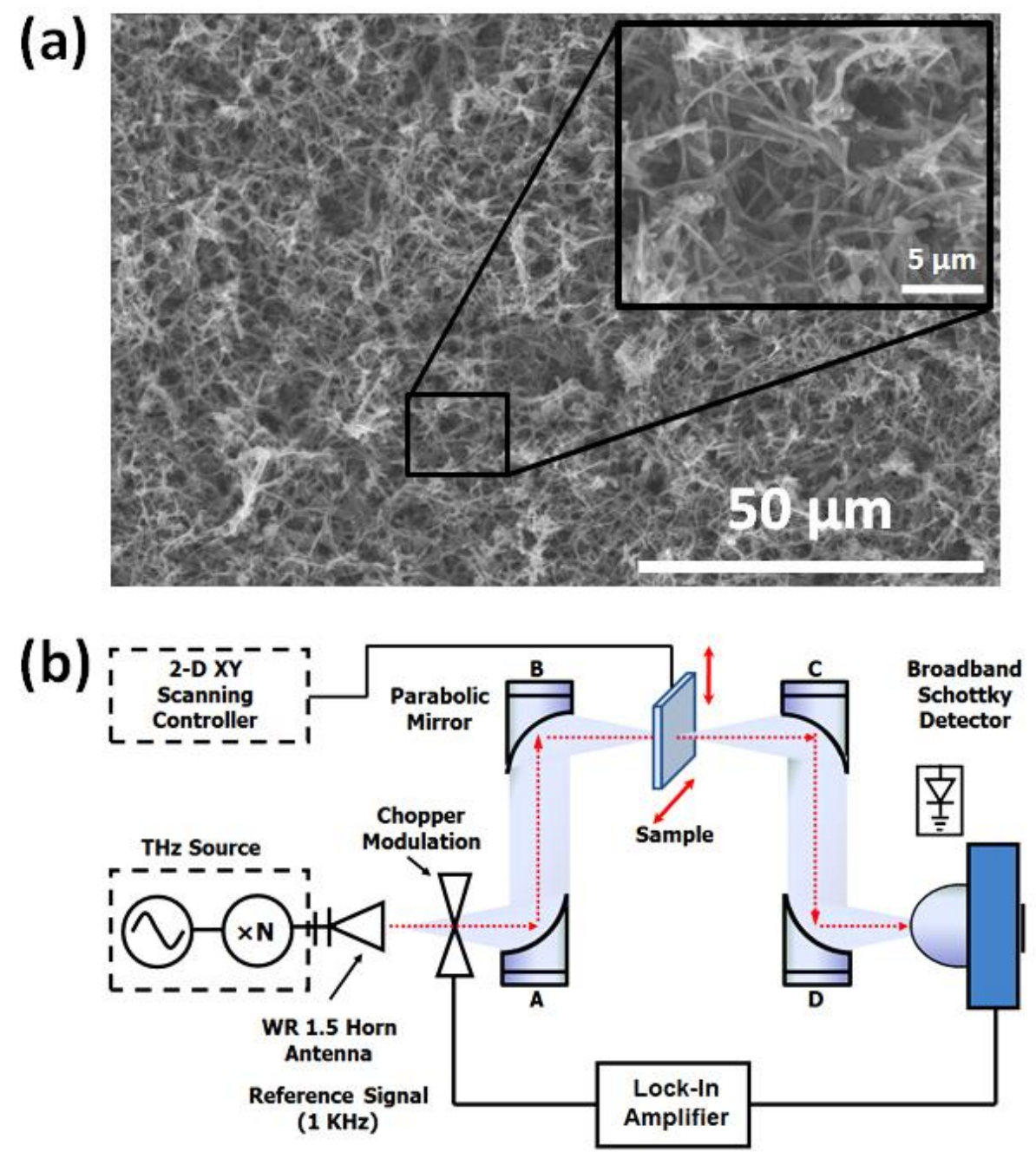

FIG. 1. (a) Scanning electron micrograph of a CNW/PMC (1:1 mass ratio) coating. (b) A frequency domain terahertz spectroscopy system based on broadband quasi-optical Schottky diode detectors. Mirrors A and B collimate and then focus the beam through the sample. Mirrors C and D collimate the transmitted signal again and focus it onto the detector. The sample under test, mounted on a computercontrolled XY positioning stage, is placed at the focal point of the THz beam between mirrors B and C. The system is designed to have a Gaussian beam waist (spatial resolution) of approximately $1 \mathrm{~mm}$ at the sample. 


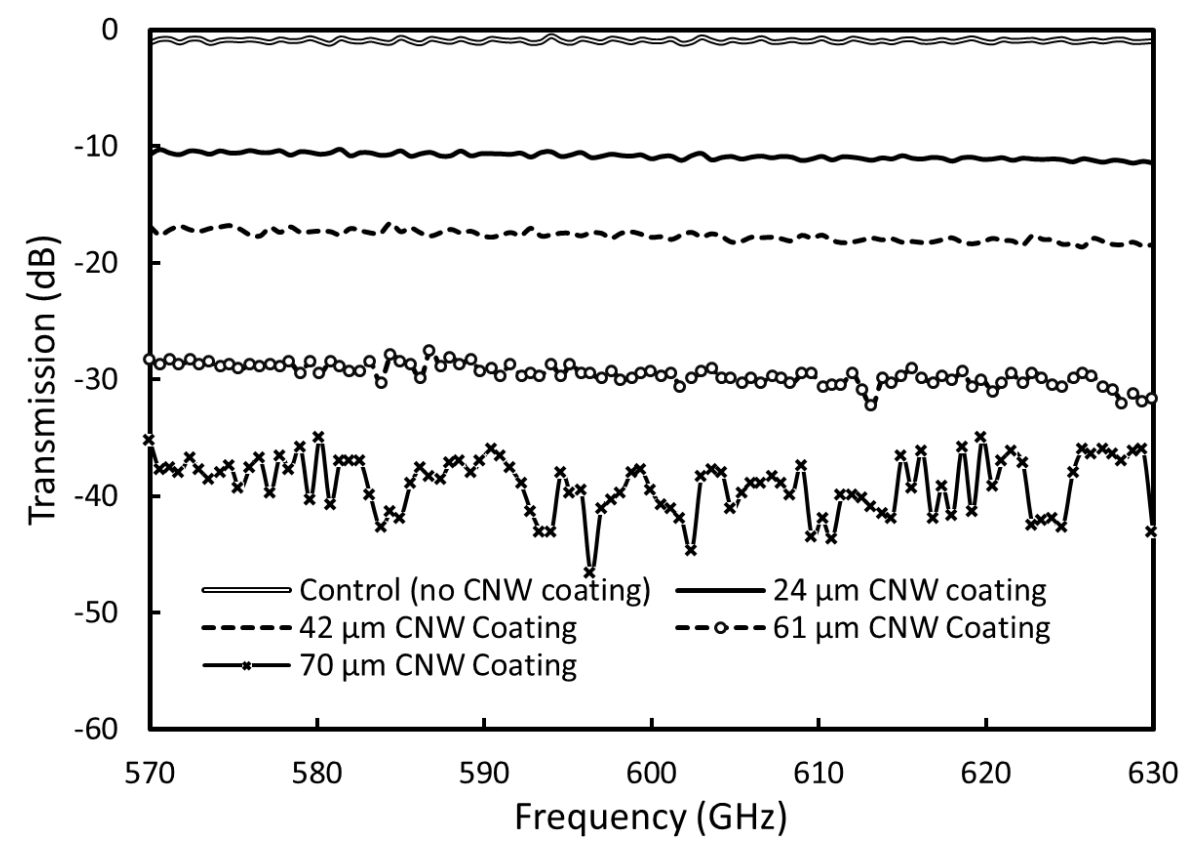

FIG. 2. THz power transmission measurement results for composite coating samples with CNW/PMC mass ratio of 1:1 and average thicknesses as stated on the legend.

Figure 2 shows the THz power transmission measurement results using the frequency domain THz system described above (Fig. 1b) for five CNW/PMC coating samples with thickness varying from $0 \mu \mathrm{m}$ (control, no $\mathrm{CNW}$ coating) to $70 \mu \mathrm{m}$. The control curve (uncoated paper) is indistinguishable from that of another sample (not shown in Fig. 2) coated with PMC only, thus confirming that transmission losses through the CNW/PMC composite coatings are attributed entirely to the CNWs. The THz transmittance (in $\mathrm{dB}$ ), or EMI SE is defined by SE $(\mathrm{dB})=-10$ $\log _{10}\left(P_{\text {tran }} / P_{\text {inc }}\right)$, where $P_{\text {tran }}$ and $P_{\text {inc }}$ denote transmitted and incident $\mathrm{THz}$ powers. In these measurements, the Schottky detector worked in the square-law region, therefore the output voltage response was proportional to the power detected. For the five samples, a gradually increasing average $\mathrm{SE}$ of $\sim 1.0 \mathrm{~dB}$ (control, no $\mathrm{CNW}$ coating) to $\sim 40 \mathrm{~dB}$ (for the thickest coating) 
is shown in Fig. 2 in the frequency range of $570-630 \mathrm{GHz}$, which is consistent with the increasing coating thickness of these samples. The transmittance curves for the first four samples are quite uniform over the entire frequency range in Fig. 2. The fluctuations observed for the 70 $\mu \mathrm{m}$-thick coating (bottom curve) are attributed to measurement noise when the transmitted $\mathrm{THz}$ power is significantly low. We also measured the SE of a double-sided sample with a $70 \mu \mathrm{m}-$ thick coating on each side. The THz SE of this sample far exceeded the system's dynamic range, thus suggesting attenuations much higher than $40 \mathrm{~dB}$, and in turn, promising extraordinary performance of $\mathrm{THz}$ polarizers printed with this formulation and at double thickness.
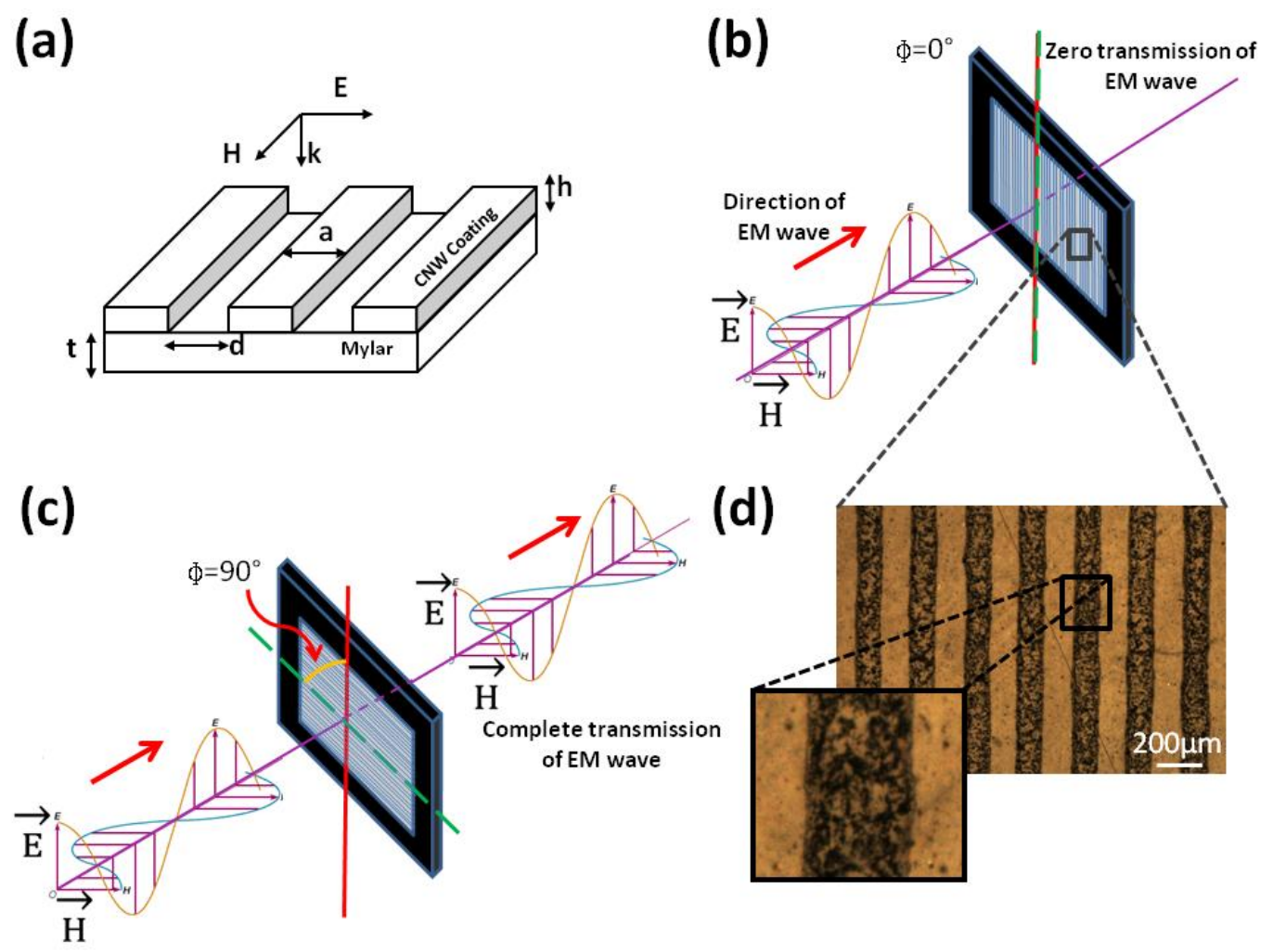

FIG. 3. (a) Design of the $\mathrm{THz}$ polarizer fabricated on $25 \mu \mathrm{m}$-thick Mylar film, with $100 \mu \mathrm{m}$-wide microstrips separated by $100 \mu \mathrm{m}$ spacing. The prototype polarizer had $\sim 1 \mu \mathrm{m}$ CNW/PMC film thickness (h), and was characterized in the frequency band of $0.57-0.63 \mathrm{THz}$ with the frequency domain $\mathrm{THz}$ spectrometer of Fig. 1b. (b) Parallel orientation (or $0^{\circ}$ ) for minimum transmission, and (c) perpendicular (or $90^{\circ}$ ) orientation for minimum attenuation. (d) Optical microscope image of the inkjet-printed 
prototype $\mathrm{THz}$ polarizer. The dark lines consist of the CNW/PMC film material. The magnified detail shows the spatial non-uniformity of a typical strip. The dark areas depict high content of CNWs, while the bright areas indicate lower particle presence.

As shown in Fig. 3a, the prototype polarizer was designed on $\mathrm{t}=25 \mu \mathrm{m}$ thick Mylar film for operation at $600 \mathrm{GHz}$. Mylar thin films were chosen as polarizer substrates because of their low insertion loss (i.e., $\sim 0.35 \mathrm{~dB}$ for $25 \mu \mathrm{m}$ thickness according to our measurement) at $\mathrm{THz}$ frequencies. Both the width (a) and spacing (d) of the composite strips are $100 \mu \mathrm{m}$, which is roughly $1 / 5$ of the wavelength ( $\sim \lambda_{0}$ free space wavelength, assuming thin substrate) at $600 \mathrm{GHz}$. The printed dispersion was made by adding CNWs in acetone and bath sonicating for 10 min. Next, acetic acid was introduced into the suspension and the bath sonication was continued for another $10 \mathrm{~min}$. Finally, $20 \mathrm{wt} \% \mathrm{PMC}$ in water was added to the suspension until the CNW/PMC mass ratio reached unity (as in the continuous coatings characterized earlier); sonication was maintained for an additional $10 \mathrm{~min}$. The final dispersion was diluted with water to a level such that the collective concentration of CNWs and PMC in the solution was less than or equal to 1.5 wt.\%. Ink jetting was performed by a MicroFab JetLab ${ }^{\circledR} 4$ XL system equipped with a $60 \mu \mathrm{m}$ diameter nozzle. The printing was performed in the drop-on-demand mode at a dispensing frequency of $500 \mathrm{~Hz}$. The device consistently generated monodisperse droplets with diameters in the range $70-75 \mu \mathrm{m}$. The spot pitch was maintained constant at $40 \mu \mathrm{m}$, translating into a printing line speed of $20 \mathrm{~mm} \mathrm{~s}^{-1}$. The distance of the device orifice from the substrate was $0.5 \mathrm{~mm}$. During the inkjet printing process, the designed pattern was printed one time to produce a coating thickness (h) close to $1 \mu \mathrm{m}$. This thickness estimate was made based on the content of each droplet, the droplet pitch, the line width and the densities of each constituent (PMC $1.5 \mathrm{~g} / \mathrm{cm}^{3}, \mathrm{CNW} 2.3 \mathrm{~g} / \mathrm{cm}^{3}$, acetone $0.79 \mathrm{~g} / \mathrm{cm}^{3}$, acetic acid $\left.1.05 \mathrm{~g} / \mathrm{cm}^{3}\right)$. Thicker coatings for better 
performance can be achieved by repeating the same process more times. Figure 3(d) shows an optical microscope image of a typical inkjet-printed THz polarizer on Mylar film. Although not perfectly produced, well-aligned CNW composite coating strips with designed width (100 $\mu \mathrm{m})$ and interspacing $(100 \mu \mathrm{m})$ were observed, demonstrating the capability of printing $\mathrm{THz}$ quasioptical components without complex fabrication procedures. In addition, an inkjet printing resolution of less than $50 \mu \mathrm{m}$ is possible with current inkjet printing techniques. Linearly patterned CNW samples as shown in Fig. 3(a) are expected to show anisotropic EM wave polarization properties in analogy to single walled nanotubes (SWNT) ${ }^{20}$ As shown in Fig. 3(c), when the E-field of the incident $\mathrm{THz}$ wave is orthogonal to the CNF strip lines $\left(\Phi=90^{\circ}\right)$, the induced magnetic currents (due to H-field) are not affected and re-radiate to the other side of the polarizer, causing minimum insertion loss. However, when the polarizer is rotated parallel (or $\Phi$ $=0^{\circ}$ ) to the E-field (see Fig. 3(b)), the polarizer allows minimum transmission. 


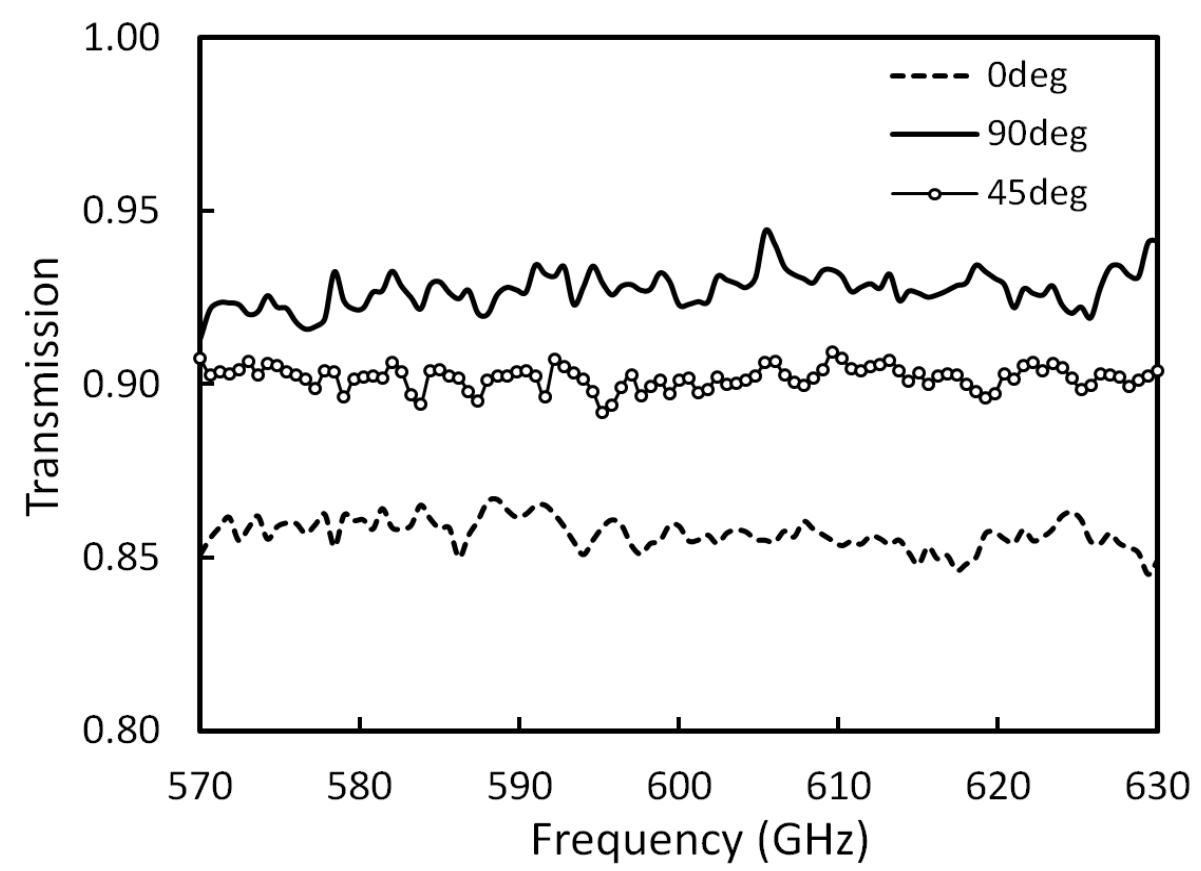

(a)

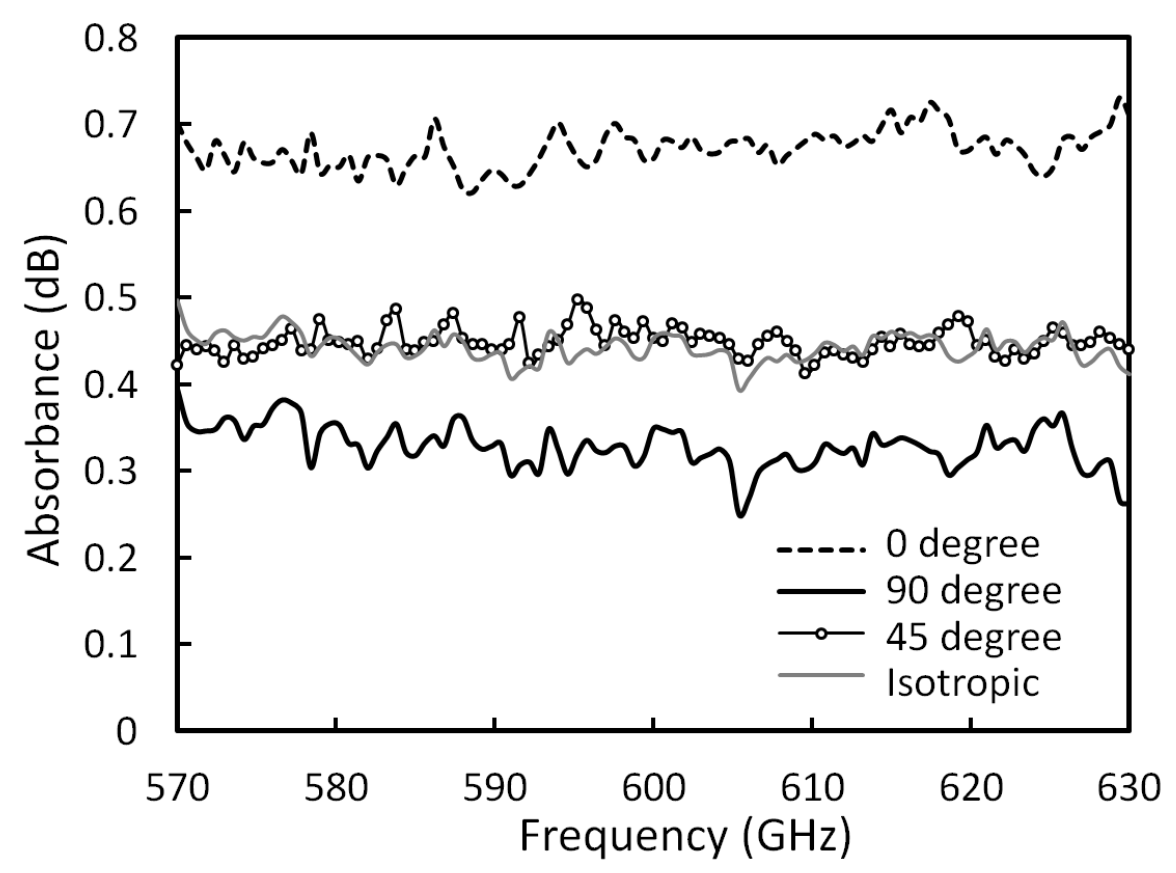

(b)

FIG. 4. (a) THz transmission (T) spectra of the prototype CNW polarizer for three different polarization orientations: $0^{\circ}, 45^{\circ}$ and $90^{\circ}$ (see Fig. 3 (c) and (d)). (b) Corresponding absorbances, defined as $\mathrm{A}=$ $-\log _{10}(\mathrm{~T})$, for the above three orientations and following Ref. [11]. 
The inkjet-printed polarizer prototype was measured in the range of $570-630 \mathrm{GHz}$ using the frequency domain THz system described previously. As shown in Fig. 4(a), the polarizer placed at perpendicular orientation $\left(90^{\circ}\right)$ showed maximum transmission averaging $\mathrm{T}_{\perp}=0.93$, while the parallel orientation $\left(0^{\circ}\right)$ gave the lowest average transmission $\mathrm{T}_{/ /}=0.86$. A $45^{\circ}$-oriented polarizer recorded a transmission level in between of $\mathrm{T}_{\perp}$ and $\mathrm{T}_{/ /}$, as expected. The observed fluctuations in these plots are attributed to system noise and minor artifacts not fully removed in our tests. Nonetheless, the fluctuations in Fig. 4(a) are small enough (within $\pm 1 \%$ ), showing fairly high measurement accuracy. To ensure that the observed anisotropic phenomena were indeed introduced by the aligned CNW microstrips, the corresponding absorbances, defined as $\mathrm{A}=-$ $\log _{10}(\mathrm{~T})$ were calculated as in Ref. [11]. Figure 4(b) displays the calculated absorbance for $0^{\circ}$ (parallel), $45^{\circ}$ and $90^{\circ}$ (perpendicular) orientations. The isotropic absorbance, which corresponds to randomly oriented CNW microstrips, was calculated as $\left(\mathrm{A}_{/ /}+2 \mathrm{~A}_{\perp}\right) / 3$ and is plotted in Fig. 4(b) using the grey line. It is observed that the isotropic curve agrees very well with the values at $45^{\circ}$, indicating that the prototype polarizer works as intended. For all the data in Fig. 4(b), the $25 \mu \mathrm{m}$ Mylar film substrate contributed only $\sim 0.35 \mathrm{~dB}$ to the absorbances. Finally, the degree of polarization $(\mathrm{P})$ of the $\mathrm{THz}$ polarizer was calculated using $\mathrm{P}=\left(\mathrm{A}_{/ /}-\mathrm{A}_{\perp}\right) /\left(\mathrm{A}_{/ /}+\mathrm{A}_{\perp}\right)$, and is plotted in Fig. 5. An average value of $P=0.32$ was measured over the entire frequency range of 570-630 GHz. The insertion loss (defined as $10 \log \left(\mathrm{T}_{\perp}\right)$ ) of the polarizer with incident wave polarization perpendicular to the alignment axis (ideally maximum transmission) decreases with decreasing values of a and d (Fig. 3a), while the fractional power transmission with incident wave polarization parallel to the composite strip direction (ideally minimum transmission) increases with $(\mathrm{a}+\mathrm{d}) / \lambda_{0} .{ }^{21}$ Thus, the performance of the polarizer shown in Fig. 3a including extinction ratio $\left(\mathrm{T}_{\perp} / \mathrm{T}_{/ /}\right)$and degree of polarization $(\mathrm{P})$ could be greatly enhanced by using finer grids with 
smaller dimensions a and d. Compared to an ideal polarizer having a unit degree of polarization, this sample shows relatively low $\mathrm{P}$ and extinction ratio. However, the prototype has been proven to work as a polarizer, and its performance can be improved by increasing the composite coating thickness and decreasing both the composite strip width (a) and spacing (d). For example, Fig. 2 shows that an extinction ratio well over $30 \mathrm{~dB}$ can be realized with a coating thickness of $70 \mu \mathrm{m}$. In addition, a dual-layer polarizer structure could be adopted to further improve the polarizer performance, and as a result, a $60-\mathrm{dB}$ extinction ratio is attainable near $600 \mathrm{GHz}$ for a polarizer with dual-layer (70 $\mu \mathrm{m}$ each) coatings, as evidenced by the CNW composite coating THz EMI properties demonstrated in Fig. 2. Such levels of extinction far exceed the recently achieved ${ }^{22}$ values of $37 \mathrm{~dB}$ in the range $0.1-2 \mathrm{THz}$ by winding nanotube sheets on U-shaped polymer frames and stacking them in 75 layers.

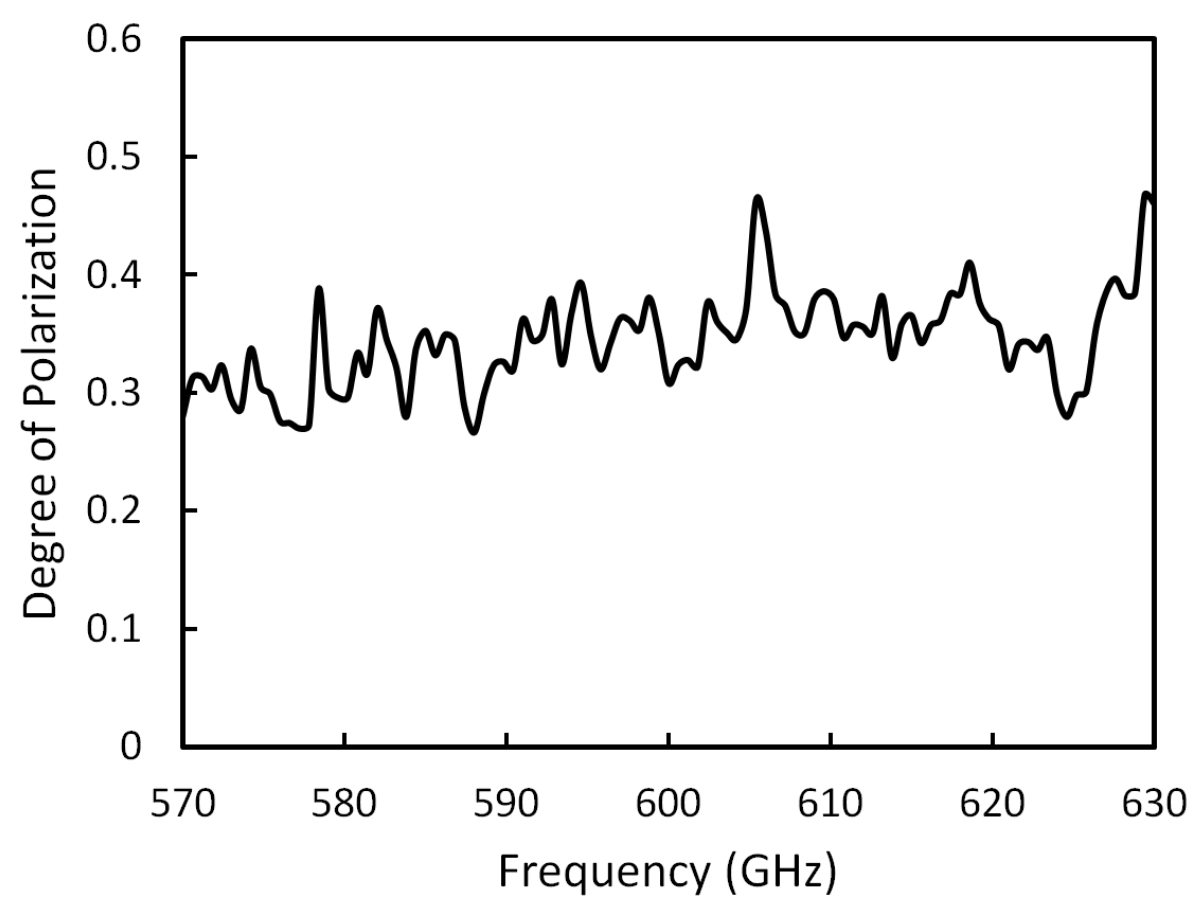

FIG. 5. The degree of polarization $(\mathrm{P})$ of the prototype THz polarizer over the frequency range of 570-630 GHz. An average degree of polarization of $\sim 0.35$ is seen for this polarizer with coating thickness of only one micron. 
In conclusion, we have developed a new approach for cost-effective manufacturing of $\mathrm{THz}$ quasi-optical polarizers based on inkjet printing of CNW/polymer composite coatings. The EMI properties of large-area CNW/PMC coatings have been studied in the frequency range 570-630 $\mathrm{GHz}$ using a frequency domain terahertz spectrometer. Shielding effectiveness of $\sim 40 \mathrm{~dB}$ has been achieved in the above bands with $70 \mu$ m-thick coatings, defining the feasible polarizer extinction ratio level. A prototype polarizer was designed and inkjet-printed on Mylar thin film with CNW composite coatings for operation near $600 \mathrm{GHz}$. The measured transmission and absorbance vary with polarization orientation. The degree of polarization of this sample was 0.3 0.4 over the entire frequency range of $570-630 \mathrm{GHz}$, for a polarizer coating thickness of only $\sim 1 \mu \mathrm{m}$. The polarizer performance can be improved by using finer grid dimensions, increasing coating thickness and adopting dual-layer polarizer structures. The results and method reported in this paper serve as a proof of concept for approaching a variety of cost-effective quasi-optical THz components such as attenuators, polarizers and filters.

\section{Acknowledgement}

The authors would like to thank Drs. P. Fay and H. Xing in the Department of Electrical Engineering, University of Notre Dame for valuable discussions. This work was partially supported by NSF Grants ECCS-1002088 and ECCS-1102214. The authors also would like to acknowledge partial supports from the Advanced Diagnostics and Therapeutics (AD\&T) and the Center for Nano Science and Technology (NDnano) at the University of Notre Dame. 


\section{References}

1 Siegel, P. H. IEEE Trans. Microwave Theory Tech. 50, 910 (2002).

2 Globus, T. R., Norton, M. L., Lvovska, M. I., Gregg, D. A., Khromova, T. B., Gelmont, B. L. IEEE Sensors Journal 10, 410 (2010).

3 Brown, E. R., Mendoza, E. A., Xia, D., Brueck, S. R. J. IEEE Sensors 10, 755 (2010).

4 Phillips, T. G., Keene, J. Proceedings of the IEEE 80, 1662 (1992).

5 Liu, L., Xu, H., Lichtenberger, A. W., Weikle II, R. M. IEEE Trans. Microwave Theory Tech. 58, 1943 (2010).

6 Gommans, H. H., Alldredge, J. W., Tashiro, H., Park, J., Magnuson, J., Rinzler, A. G. J. Appl. Phys. 88,2509 (2000).

7 Bird, G. R., Parrish, M. J. Opt. Soc. Am. 50, 875 (1960).

8 Zhang, L., Teng, J. H., Tanoto, H., Yew, S. Y., Deng, L. Y., Chua, S. J., "Terahertz wire-grid polarizer by nanoimprinting lithography on high resistivity silicon substrate," The International Conference on Infrared, Millimeter, and Terahertz Waves, Rome, Italy, 2010.

9 Yamada, I. Optics Lett., 34, 274 (2009).

10 Tian, D.-B., Zhang, H.-W., Lai, W.-E., Wen, Q.-E., Song, Y.-Q., Wang, Z.-G. Chin. Phys. Lett. 27, 104210 (2010).

11 Ren, L., Pint, C. L., Booshehri, L. G., Rice, W. D., Wang, X., Hilton, D. J., Takeya, K., Kawayama, I., Tonouchi, M., Hauge, R. H., Kono, J., Nano Lett. 9, 2610 (2009).

12 Kyoung, J., Jang,, E. Y., Lima, M. D., Park, H.-R., Robles, R. O., Lepro, X., Kim, Y. H., Baughman, R. H., Kim, D.-S., Nano Lett., 11, 4227 (2011).

13 Das, A., Megaridis, C. M., Liu, L., Wang, T., Biswas, A. Appl. Phys. Lett. 98, 174101 (2011).

14 Orecchini, G., Alimenti, F., Palazzari, V., Rida, A., Tentzeris, M. M., Roselli, L. IET Microw. Antennas Propag. 5, 993 (2011).

15 Shaker, G., Safavi-Naeini, S., Sangary, N., Tentzeris, M. M. IEEE Antennas and Wireless Propagation Letters 10, 111 (2011).

16 Deegan, R.D., Bakajin, O., Dupont, T. F., Huber, G., Nagel, S. R., Witten, T. A. Nature 389, 827 (1997).

17 Yunker, P. J., Still, T., Lohr, M. A., Yodh, A. G., Nature 476, 308 (2011).

18 Liu, L., Hesler, J. L., Weikle, R. M., Wang, T., Fay, P., Xing, H., International Journal of High Speed Electronics and Systems 20, 629 (2011). 
${ }^{19}$ Liu, L., Hesler, J. L., Xu, H., Lichtenberger, A. W., and Weikle, R. M., IEEE Microwave and Wireless Components Letters 20, 504 (2010).

${ }^{20}$ Jorio, A., Dresselhaus, G., Dresselhaus, M. S., Eds., "Carbon Nanotubes: Advanced Topics in the Synthesis, Structure, Properties and Applications," Springer:Berlin, 2008.

${ }^{21}$ P. F. Goldsmith, "Quasioptical Systems: Gaussian Beam Quasioptical Propagation and Applications," IEEE Press/Chapman \& Hall Publishers Series on Microwave Technology and Techniques, 1997.

22 Kyoung,J., Jang, E. Y., Lima, M. D., Park, H.-R., Robles, R. O., Lepro, X., Kim, Y. H., Baughman, R. H., Kim, D.-S., Nano Letters 11, 4227 (2011). 